\title{
Rúbrica de autoevaluación para promover la competencia argumentativa en foros de discusión en línea
}

\section{Promoting argumentative competence by self-assessment rubric in online discussion forums}

\author{
Yunuen Ixchel Guzmán-Cedillo ${ }^{1}$ \\ Facultad de Psicología \\ Universidad Nacional Autónoma de México \\ México D.F. \\ yunuenixchel@hotmail.com
}

\begin{abstract}
Recibido: 3 diciembre 2012 Aceptado: 12 Julio 2013 Corregido: 7 Noviembre 2013
\end{abstract}
Resumen: El objetivo de esta investigación es determinar diferencias en los niveles de desempeño de la competencia argumentativa en foros de discusión a partir del uso de rúbricas por parte de los y las estudiantes inscritos en la materia en línea Sexualidad humana. El diseño fue cuasi experimental longitudinal tipo panel. Tres codificadores evaluaron los mensajes escritos por los participantes en tres foros de discusión (7, 16 y 21), en uno de ellos (número 16) se implementó la rúbrica de autoevaluación para los y las estudiantes. Las participaciones fueron evaluadas y ubicadas en un nivel de desempeño (del 1 al 4), los cuales se compararon a través de una prueba de rangos de Wilcoxon para determinar diferencias. Los resultados sugieren que el uso de rúbricas de autoevaluación apoya en cuatro de los cinco elementos contenidos en la rúbrica.

Palabras clave: Rúbrica, autoevaluación, discusión en línea, argumentación, competencias.

Abstract: The objective of this paper is to determine differences on performance levels when students use a self-assessment rubric into online discussion forums as a tool to write a good message. This educational experience was in online forums about human sexual education for medical students. A quasi-experimental research design was developed. In one forum (16) students use a rubric for writing their messages, their performance level was compare to levels from other forums (7,16 y 21) through Wilcoxon test analysis. Results suggest that using a self-assessment rubric

promotes argumentative competence in four of five rubric's elements. Writing messages is a complex activity where different abilities are mixed at the same time.

Key words: rubrics, self-assessment, online discussion, reasoning, skills/competences.

Candidata a Doctora por el Programa de la Universidad Nacional Autónoma de México (UNAM). Maestría en Psicología por la Facultad de Psicología Universidad Nacional Autónoma de México (UNAM). Licenciatura en Psicología por la Facultad de Estudios Superiores Iztacala (FESI) UNAM. Se ha desempeñado como docente en diferentes niveles educativos desde secundaria hasta posgrado, tanto en la formación presencial como virtual en escuelas públicas y privadas, establecimientos como el Instituto Sud Médoc (Ministerio de educación, Burdeos, Francia), la Universidad Tecnológica de México (UNITEC), la Universidad Iberoamericana (UIA), la Escuela Nacional de Medicina y Homeopatía del Instituto Politécnico Nacional (ENMyH), el bachillerato en línea de la UNAM, y actualmente colaboro como supervisora en sede en el programa de maestría con residencia en psicología escolar de la Facultad de Psicología de la UNAM y como coordinadora de diseño curricular en la Universidad del Valle de México (UVM). Sus intereses en investigación educativa y desarrollo profesional han girado sobre temas diversos como son: el papel del docente en la detección de maltrato infantil, aprendizaje cooperativo en el aprendizaje de una segunda lengua, la formación psicológica del médico, la formación profesional desde la perspectiva de competencias, desarrollo de rúbricas como formas de evaluación de competencias, capacitación docente en el enfoque de competencias y entornos virtuales de aprendizaje. Estos trabajos han tenido diferentes escenarios de difusión como los congresos y coloquios organizados por el COMIE, CENEIP, SOMECE, MATI (Montreal), Gabinete de comunicación de la Universidad de Barcelona, entre otros. 


\section{Los foros de discusión en línea}

Las tecnologías generan espacios de comunicación óptimos para la formación de profesionales dentro de modalidades educativas completamente a distancia o híbridas. Específicamente la argumentación en procesos de formación en línea ha sido objeto de estudio por sus implicaciones para comunicar ideas y adquirir conocimiento, al desarrollar un pensamiento científico.

En esta investigación se estudió como espacio de intercambio comunicativo a los foros de discusión en línea, los cuales refieren un tipo de comunicación mediada por computadora (CMC) parecida a una conversación, donde los mensajes son aportaciones escritas ordenadas en turnos etiquetados con un nombre para que quienes participan sepan de qué trata su comentario (véase figura 1).

Los foros de discusión se utilizan de diferente manera y grado en procesos de formación en línea, pues sirven para la interacción social, para la discusión de tareas u otros trabajos de evaluación, para negociar la toma de decisiones en un equipo de trabajo o para realizar una estrategia educativa (Guzmán, Flores y Tirado, 2012).

En concreto las dos características principales del foro de discusión son:

1) es una forma de comunicación escrita de tipo asincrónico para realizar el intercambio a la vez que rescata aspectos de la conversación oral como es el uso de turnos y,

2) la interacción puede ocurrir entre varios participantes o de uno a uno, frecuentemente iniciada con un mensaje detonador que invita a la deliberación alrededor de un tema (Veerman, Andriessen, y Kanselaar, 2000; Coffin y Hewings, 2005).

Cuando se considera a los foros de discusión en línea como oportunidades de desarrollo de competencias argumentativas, también se plantea que los miembros del grupo pueden en esos espacios dar una respuesta pensada, gracias a la permanencia del diálogo, a la posibilidad de hacer una aportación en cualquier momento y a la igualdad entre los miembros para poder participar (efecto de participación igualitaria); las personas se sienten menos cohibidas al interactuar porque hay menos posibilidades de anteponer un estatus entre los participantes del grupo (Guzmán-Cedillo, 2013).

Por su parte Coffin y Hewings (2005), observaron que 47 estudiantes de una maestría en lingüística, seleccionaron de manera más cuidadosa la evidencia a sus contribuciones en foros de discusión en línea que cuando realizaban ensayos escritos. Explicaron los resultados a partir del compromiso de los estudiantes en el diálogo realizado, donde los participantes tenían una expectativa de intercambio a diferencia del ensayo escrito, debido a que tomaban tiempo de pensar antes de escribir una réplica a un interlocutor.

A su vez, Hammond (2000), encontró que los estudiantes refirieron sentimientos positivos de motivación y creatividad, a través de un estudio de casos múltiples sobre la percepción de la potencialidad del uso de foros de discusión en línea en el aprendizaje, también encontraron agradable la comunicación asíncrona debido a que les permitió ver sus mensajes y los de sus compañeros en el momento que ellos decidían (son libres de la imposición de horarios o la 
demanda de atención de personas que hablan o escriben rápido, de los ambientes presenciales o del chat).

En esas investigaciones se subraya el hecho de describir a los foros de discusión en línea como una discusión lenta que ofrece a los participantes tiempo considerable para reflexionar y ponderar sus intervenciones. En el caso de foros académicos, estos se convierten en escenarios de intercambio de información: artículos, videos o escritos estructurados realizados por los participantes que someten al escrutinio de sus compañeros.

\section{La competencia argumentativa}

Cuando el foro es comprendido como una situación que permite la libre expresión de pensamientos con el objetivo de explicar un razonamiento a otros interesados en la temática o problema a revisar, se genera un espacio ideal para estudiar la competencia argumentativa (Fu-Ren, Lu-Shi y Fu-Tai,. 2009, Hammond y Wirinapinit, 2005; Kanuka , Rourke, y Laflamme, 2007; Kear, 2001; Khun, Iordanou, Pease,y Wirkala,2008; Mason y Scirica, 2006; Lewis, 2005; Nussbaum, Sinatra y Poliquin, 2008; Veerman, Andriessen, y Kanselaar, 2000; Ying-Hua, 2006).

El proceso de construir un argumento es en sí mismo una situación compleja que demanda una movilización de recursos. Este proceso de elaboración es aún más desafiante cuando se da en foros de discusión en línea, ya que para lograr la comprensión de otros y compartir significados se debe lograr un diálogo. Además de poner en funcionamiento las habilidades de lectura, negociación, debate, intercambio, pensamiento crítico, comunicación, razonamiento, paciencia y reflexión, en una misma situación.

Con ese contexto se entiende la competencia argumentativa como la capacidad de exponer de manera coherente, fundamentada y sistemática una afirmación, haciendo un posicionamiento frente a la temática, al mismo tiempo que se muestra una actitud frente a los interlocutores, en una situación donde se intercambian y comparten significados (GuzmánCedillo, 2013).

Con el objeto de fortalecer los recursos importantes en los mensajes de foros académicos, se determinó que el lenguaje (vocabulario disciplinar), la fundamentación (tipo de evidencia), la colaboración (intercambio), el respeto (consideración) y la contraargumentación (responder a los interlocutores), conforman una articulación competente al escribir un mensaje en foros de discusión.

Dichos recursos son definidos en los siguientes párrafos:

- Lenguaje: se refiere al manejo de nociones conceptuales que muestran profundidad en el conocimiento de un tema.

- Fundamentación: puntualiza la evidencia enunciada, si hay ausencia, si existe una generalización basada en la experiencia o si se cita una fuente legitimada.

- Colaboración: hace referencia a las actitudes que permiten la invitación al debate 
y la deliberación así como el compromiso asumido en una argumentación dialógica referente a la disposición o no a debatir con los interlocutores.

- Respeto: capacidad para manifestar desacuerdos, cuando se es asertivo y se muestra al declarar inconformidad.

- Contraargumentación: mensajes con los cuales se responde una aceptación o negación de la fiabilidad de un argumento, desechándolos total o parcialmente.

- Al comprender que la competencia argumentativa pone en juego una serie de recursos coordinados en una ejecución cuyo desarrollo ocurre gradualmente, se vuelve pertinente realizar la evaluación del desempeño de esta competencia por medio de rúbricas ya que esta forma de valoración contempla varios indicadores que diferencian distintos niveles de desarrollo y dimensiones (recursos).

\section{La rúbrica de autoevaluación}

Asimismo se ha observado que las habilidades de los participantes para elaborar un argumento mejoran a partir de la exposición a criterios de un buen argumento científico contenidos en rúbricas de autoevaluación (Nussbaum, Sinatra y Poliquin, 2008; Mason y Scirica, 2006).

De manera general, la rúbrica se entiende como una matriz de valoración de desempeño en actividades que son complejas a partir de un conjunto de criterios graduados. Cuando se valora la articulación de diferentes recursos como una capacidad de actuar en una situación dada, la rúbrica cualifica de manera progresiva el tránsito de un desempeño de novato a experto al evidenciar un nivel competente en términos de la presencia de elementos juzgados como esenciales y que permiten emitir un juicio sobre el valor global del desempeño (Cazares y Cuevas, 2008; Díaz -Barriga, 2006; Tobón, Rial, Carretero y García, 2006).

Frecuentemente las rúbricas son representadas en forma de una rejilla o tabla que incluyen criterios graduados enlistados en niveles de ejecución desde lo más básico hasta llegar a los más expertos o complejos (véase figura 1). La función de los criterios es proveer de una medida general para todo el producto o ejecución esperados que se muestra en toda la rúbrica, y al mismo tiempo se dan medidas particulares a través de los elementos esenciales (rubros) o dimensiones que pueden ser valoradas de forma separada (Okleaf, 2009). 


\begin{tabular}{|c|c|c|}
\hline \multicolumn{3}{|c|}{ Rúbrica de calidad de argumentos, contrargumentos y refutaciones } \\
\hline Puntaje & Descripción & Ejemplos \\
\hline 0 & $\begin{array}{l}\text { No hay argumento pues no se } \\
\text { provee de justificaciones. }\end{array}$ & $\begin{array}{l}\text { "estoy en desacuerdo con él, no es correcto" "voy } \\
\text { a repetir mi explicación acerca del calentamiento } \\
\text { global" }\end{array}$ \\
\hline 1 & $\begin{array}{l}\text { No es un argumento completa- } \\
\text { mente válido }\end{array}$ & $\begin{array}{l}\text { "pienso que los productos OGM debe ser marcado } \\
\text { como buenos productos" }\end{array}$ \\
\hline 2 & $\begin{array}{l}\text { Argumento válido pero con } \\
\text { pocos razonamientos. }\end{array}$ & $\begin{array}{l}\text { "el calentamiento global no se debe a la actividad } \\
\text { humana porque también hace } 1000 \text { o } 1500 \text { años había } \\
\text { un incremento de la temeratura" }\end{array}$ \\
\hline 3 & $\begin{array}{l}\text { Argumento válido que da un } \\
\text { razonamiento. }\end{array}$ & $\begin{array}{l}\text { "creo que está bien producir alimentos transgénicos } \\
\text { porque los insectos que le hacen daño son muy resis- } \\
\text { tentes y hacen daño a las cosechas" }\end{array}$ \\
\hline 4 & $\begin{array}{l}\text { Argumento válido que da dos o } \\
\text { más razonamientos }\end{array}$ & $\begin{array}{l}\text { "podría comentar que no estoy de acuerdo con la } \\
\text { generación de alimentos transgénicos por que no han } \\
\text { mostrado combatir la hambruna ya que este problema } \\
\text { tiene un origen económico y político que viven los } \\
\text { países pobres en cuanto a la desigualdad de repar- } \\
\text { tición de los recursos y no por problemas de agricul- } \\
\text { tura..." }\end{array}$ \\
\hline
\end{tabular}

Figura 1. Ejemplo de rúbrica de evaluación

Traducida y adaptada de Mason y Scirica (2006, p. 499)

En este sentido, una función fundamental de la rúbrica es guiar a los estudiante en el análisis de procesos de aprendizaje a través de una descripción detallada de los niveles de ejecución, es decir, se puntualiza qué se espera de ellos a partir de lo cual se emiten juicios sobre la calidad de sus tareas que promueve la propia evaluación (Etkina, Van Heuvelen, WhiteBrahmia, Brookes, Gentile, Murthy, Rosengrant y Warren, 2006; Gürsul y Keser, 2009).

De manera general, se entiende la rúbrica como una matriz de valoración de desempeño en actividades que son complejas a partir de un conjunto de criterios graduados. La función de los criterios es proveer de una medida general para todo el producto o ejecución esperados que se muestra en toda la rúbrica, y al mismo tiempo se dan medidas particulares a través de los elementos esenciales (rubros) o dimensiones que pueden ser valoradas de forma separada (Endrizzi y Rey, 2008; Oakleaf, 2009).

En una investigación de los beneficios que significan las rúbricas, Jonsson y Svingby (2007) mencionan que estos instrumentos tienen el potencial de promover el aprendizaje y la instrucción. La razón principal de ello es que hacen explícitos los criterios de evaluación, lo que facilita la retroalimentación y la autoevaluación (Etkina et al., 2006).

En ese sentido las rúbricas son una forma de evaluación viable para valorar la competencia argumentativa además de ser herramientas potenciales para los participantes en la elaboración de formas más complejas de argumentación en sus participaciones dentro de foros de discusión en línea.

Dada su relevancia, el presente trabajo se centra en el estudio de la competencia argumentativa dentro de foros de discusión de un proceso de formación profesional en línea. 
Con base en lo anterior, el planteamiento del problema refiere a cuál es el efecto en el nivel de desempeño de la competencia argumentativa del uso de rúbricas de autoevaluación por parte de los y las estudiantes en los foros de discusión de un proceso de formación profesional en línea. Teniendo como objetivo de investigación el determinar diferencias en los niveles de desempeño de la competencia argumentativa en foros de discusión a partir del uso de rúbricas de autoevaluación por parte de los y las estudiantes inscritos en la materia en línea de Sexualidad humana de la Escuela Nacional de Medicina y Homeopatía (ENMyH).

Cuya hipótesis de investigación es: el nivel de desempeño (1-4) de los y las estudiantes en el foro 16 se incrementa con el uso de rúbricas de autoevaluación.

\section{Método}

Participantes:

11 estudiantes, quienes escribieron mensajes en todos los foros analizados inscritos en la materia en línea de Sexualidad humana.

2 evaluadores independientes, quienes ubicaron en un nivel de desempeño las participaciones de los y las estudiantes en los tres foros analizados.

Unidad de análisis:

La unidad de análisis empleada fue el contenido discursivo del mensaje, definido en los recursos de la competencia argumentativa. Se consideraron todos los mensajes realizados por 11 estudiantes en foros que contenían temas de debate (3 foros elegidos de la secuencia didáctica de la materia en línea de sexualidad humana en la plataforma Moodle de la Escuela Nacional de Medicina y Homeopatía).

Instrumentos:

Dos rúbricas, una para los evaluadores y la rúbrica de autoevaluación para los participantes (utilizada en el foro 16).

Rúbrica de evaluadores:

Matriz de doble entrada con nivel de medida ordinal (1-4), que valora el desempeño en recursos complejos a partir de un conjunto de criterios graduados. Su confiabilidad fue determinada a través de un alpha de Cronbach de 0.84 y validez de contenido determinada por jueces expertos (Guzmán-Cedillo, Flores y Tirado, 2012).

Rúbrica de autoevaluación:

Versión adaptada de la rúbrica de evaluadores y de acceso a los participantes en el foro 16 (véase material complementario 1).

Estrategias de recolección y análisis de información:

Observación: A través de la rúbrica los evaluadores ubicaron de forma independiente los mensajes de cada estudiante en un nivel de desempeño de cada categoría (lenguaje, colaboración, respeto, fundamentación y contraargumentación). 
En esta investigación se opta por un estudio cuasi experimental longitudinal tipo panel. Las participaciones del mismo grupo de estudiantes fueron evaluadas con tres mediciones del nivel de desempeño de la competencia argumentativa.

Si bien la secuencia didáctica de la materia en línea contempló 22 foros, la competencia argumentativa se evaluó en los mensajes escritos de tres foros (7, 16 y 21) por la razón de temporalidad (se tomó un foro del inicio, uno de la mitad y uno del final del curso), además en el foro 16 los y las estudiantes contaron con una rúbrica de autoevaluación como apoyo para escribir sus mensajes.

Debido a que en un análisis de discurso es fundamental dar contexto a las y los lectores, se determinó no modificar los foros de discusión. Así, después de organizar todas las conversaciones en archivos de Microsoft Word, se presentaron a dos evaluadores que utilizaron la rúbrica para valorar de forma independiente cada mensaje.

Con el objetivo de determinar el nivel de desempeño (del uno al cuatro) de cada mensaje se ubicaron los casos en los que había discrepancia, en esos casos se pidió a un tercer evaluador que revisase estos mensajes en el contexto del foro para determinar el nivel de desempeño asignando al mensaje con 2 de 3 posibilidades de acuerdo, y en el caso de que los 3 evaluadores tuviesen una discrepancia, dos de ellos determinaban de manera conjunta el nivel de desempeño que manifestaba ese mensaje (estimación unitaria o valoración compartida del fenómeno como proponen Byman, Järvela y Hakkinen, 2005; Järvela, Järvenoja y Veerman, 2005), obteniendo así la base de datos por mensaje (cuando el estudiante había escrito más de un mensaje se obtuvo la mediana para tener un nivel de desempeño representativo del estudiante por foro).

Con la sábana de datos obtenida se procedió a realizar el análisis inferencial con la prueba no paramétrica de rangos de Wilcoxón recomendada para muestras relacionadas (medidas de un mismo grupo en momentos distintos), utilizando el programa SPSS versión 13, con el objeto de determinar las diferencias entre el nivel de desempeño de la competencia argumentativa en los foros analizados.

\section{Resultados}

En esta sección se muestran los resultados obtenidos de la evaluación de los mensajes en los tres foros analizados (7,16 y 21) (lenguaje, colaboración, respeto, fundamentación y contraargumentación). Con respecto a cada foro se obtuvo un nivel representativo (mediana, porque las medidas eran parte de una escala ordinal) del desempeño en los rubros de la competencia argumentativa que se encontraban en la rúbrica de autoevaluación.

Las medianas de nivel de desempeño se sometieron a un análisis no paramétrico por medio de una prueba de rangos de Wilcoxon (para determinar a cuál foro se le atribuían diferencias en el nivel de desempeño de la competencia argumentativa), para dos muestras relacionadas acompañada de la corrección de Bonferrani, la cual menciona que cuando se hacen seis comparaciones de dos a dos la decisión deberá basarse en un nivel crítico de .083 para cuando el nivel de significancia obtenido sea $\mathrm{p} \leq .083$. 
Los resultados sugieren que el foro 16 donde se utilizó la rúbrica de autoevaluación por parte de los y las estudiantes difiere significativamente del foro 7 en los rubros de colaboración, respeto, fundamentación y contraargumentación. Con respecto al foro 21 el foro 16 no muestra diferencias significativas (véase tabla 1 ).

\section{Tabla 1}

\section{Valores $\mathrm{Z}$ y niveles de significancia (sig. asintótica bilateral) en los resultados de prueba de Wilcoxon}

\begin{tabular}{cccccc}
\hline $\begin{array}{c}\text { Foros } \\
\text { comparados }\end{array}$ & Lenguaje & Colaboración & Respeto & Fundamentación & Contraargumentación \\
\hline Z & -1.134 & -2.540 & -2.588 & -1.84 & -2.460 \\
F16-F7 & .257 & .008 & .009 & .066 & .014 \\
Z & -1.394 & -054 & -333 & -.496 & -577 \\
F21-F16 & .163 & .957 & .739 & .620 & .524 \\
\hline
\end{tabular}

El foro 16 difiere significativamente del foro 7 en los rubros de colaboración, respeto, fundamentación y contraargumentación.

Con estos datos no se acepta la hipótesis de investigación, ya que la rúbrica de autoevaluación mostró diferencias significativas en cuatro de los cinco rubros contenidos en ella. Sin embargo, se puede observar que el uso de la rúbrica de autoevaluación en el foro 16 muestra una ganancia con respecto al foro 7 en cuatro de los cinco rubros, mantenida en el foro 21 .

\section{Conclusiones}

En esta investigación la rúbrica de autoevaluación fue una variable independiente sobre el nivel de competencia argumentativa en un foro particular; no obstante como lo menciona Oakleaf (2009) la rúbrica es una herramienta de comunicación entre profesores y alumnos, así como también lo reportan Jonsson y Sivingby (2007), al describirla como un marco comprensivo que hace explícitos los criterios y expectativas de una formación facilitando así la retroalimentación y autoevaluación.

La falta de diferencias significativas en el rubro de lenguaje parece explicarse porque la rúbrica no parece ser la adecuada en este rubro para discriminar a los y las estudiantes de nivel educativo superior. En consecuencia se sugiere realizar una revisión de los criterios de desempeño contenidos para mejorar este rubro a la luz de estos resultados.

No obstante las limitantes en la rúbrica de autoevaluación, un hallazgo interesante de discutir, es que si bien su utilización no repercute de manera integral en la competencia argumentativa, sí lo hace de manera específica en las actitudes colaborativa y respetuosa 
así como en las habilidades argumentativas de fundamentación y contraargumentación. Actitudes que refieren a la forma en que los y las estudiantes entienden deben contribuir en la deliberación del foro y la consideración de las participaciones de sus compañeros, además de dar evidencia y fuerza a sus argumentos.

El valor de este resultado está relacionado con lo que Penny y Murphy (2009) subrayan en su meta-análisis sobre la necesidad de que el diseño educativo de los foros debiese proponer ideas divergentes para que los participantes sean estimulados a comentar el trabajo del otro.

$\mathrm{Al}$ respecto se puede comentar que la rúbrica tiene implicaciones en la forma en que los y las estudiantes participan en el foro, en términos de interacción (colaboración y respeto), así como en la evidencia citada al fundamentar sus mensajes o responder a los comentarios de sus compañeros (fundamentación y contraargumentación). Estos resultados apoyan lo encontrado por Sadler y Fowler (2006) sobre la potencialidad de la rúbrica como un método de ayuda para que los y las estudiantes logren una interacción importante que promueva la construcción de conocimiento.

Por último, es necesario reconocer que el enfoque de competencias acogido en educación (holístico), demanda una concepción de la competencia como un actuar responsable que descansa sobre valores sociales como lo son el compartir y la solidaridad, producto de un aprendizaje complejo y contextualizado en situaciones que permiten el desarrollo de formas innovadoras de resolución de problemas cuando los y las estudiantes son llevados a articular los valores, conocimientos y actitudes en una misma actividad (Roegiers, 2006).

\section{Referencias:}

Byman, A., Jarvela, S., y Hakkinen, P. (2005). What Is Reciprocal Understanding In Virtual Interaction? [¿Qué es comprensión recíproca en la interacción virtual?]. Instructional Science, 33(2),121-136.

Coffin, C., y Hewings, A. (2005). Engaging Electronically: Using CMC to Develop Students Argumentation Skills in Higher Education [Involucrándose electrónicamente: usando CMC para desarrollar habilidades argumentativas de los estudiantes en educación superior]. Language and Education, 19(1), 32-49.

Cazares, A., y Cuevas, F. (2008). Las competencias: un invitado sorpresa en el mundo de la educación. Planeación y evaluación basadas en competencias docentes, desde preescolar hasta posgrado. México: Trillas.

Díaz Barriga, F. (2006). La evaluación auténtica centrada en el desempeño: una alternativa para evaluar el aprendizaje y la enseñanza. En F. Díaz, Enseñanza situada: vínculo entre la escuela y la vida (pp. 125-163). México: McGrawHill.

Endrizzi, L., y Rey, O. (2008). Assessment: a central issue for learning [La evaluación un tema central para el aprendizaje]. Dossier d'actualité (ancien titre : Lettre d'information), 39, 1-17 Recuperado de http://ife.ens-lyon.fr/vst/DA-Veille/39-november-2008 EN.pdf

Etkina, E., Van Heuvelen, A., White-Brahmia, S., Brookes, D. T., Gentile, M., Murthy, S., y Warren, A. (2006). Developing and assessing student scientific abilities. Physical Review. 
Special Topics [Desarrollando y evaluando las habilidades científicas de los estudiantes]. Physics Education Research, 2, 020-103.

Fu-Ren L., Lu-Shih H., y Fu-Tai C.(2009). Discovering genres of online discussion threads via text mining [Descrubiendo géneros de los hilos de discusión vía significado del texto]. Computers \& Education, 52(2), 481-495. Recuperado de http://dl.acm.org/citation.cfm?id=1480548

Gürsul, F., y Keser, H. (2009). The effects of online and face to face problem based learning environments in mathematics education on student's academic achievement [Los efectos de la resolución de problemas cara a cara y en línea en el logro academico de estudiantes dentro de ambientes de aprendizaje en educación matemática]. Procedia-Social and Behavioral Sciences, 1(1), 2817-2824.

Guzmán-Cedillo, Y. I. (2013). Desarrollo de la competencia argumentativa en foros de discusión en línea (Tesis doctoral, Universidad Nacional Autónoma de México, México) Recuperado de http://bcct.unam.mx/web/tesiunam.htm.

Guzmán-Cedillo, Y., Flores, R., y Tirado, F. (2012). La evaluación de la competencia argumentativa en foros de discusión en línea a través de rúbricas. Revista Innovación Educativa, 12(60), 17-40. Recuperado de: http://www.redalyc.org/pdf/1794/179426856003.pdf

Hammond, M., y Wiriyapinit, M. (2005). Learning through online discussion: A case of triangulation in research [El aprendizaje a través de las discusiones en línea. un caso de triangulación en investigación]. Australasian Journal of Educational Technology, 21(3), 283. Recuperado de http://www.ascilite.org.au/ajet/ajet21/hammond.html

Hammond, M. (2000). Communication within on-line forums: the opportunities, the constraints and the value of a communicative approach. [Comunicación en foros en línea: las oportunidades, las contrariedades y el valor de la aproximación comunicativa] Computers \& Education, 35(4), 251-262.

Järvelä, S., Järvenoja, H., y Veermans, M. (2005). How students describe the sources of their emotional and motivational experiences during the learning process: A qualitative approach [¿Cómo los estudiantes describen los recursos de sus experiencias motivacionales y afectivas durante el proceso de aprendizaje: una aproximación cualitativa]. Learning and Instruction, 15, 465-480.

Jonsson, A., y Svingby, G. (2007). The use of scoring rubrics: Reliability, validity and educational consequences [El uso de rúbricas de evaluación: confiabilidad, validez y consecuencias educativas]. School of Teacher Education Educational Research Review, 2(2), 130-144.

Kanuka, H., Rourke, L., y Laflamme, E. (2007).The influence of instructional methods on the quality of online discussion [La influencia de métodos instruccionales en la calidad de las discusiones en línea].. British Journal of Educational Technology, 38(2) 260-271

Kear, K., (2001). Following the thread in computer conferences [Siguiendo el hilo en conferencias en línea]. Computers \& Education, 37, 81-99.

Khan, A., y Lawrence, R. (2006). Use of rubrics for assessment of a senior project design course [Uso de rúbricas para la evaluación de diseño de proyectos en cursos de educación superior]. 
Trabajo presentado en ASEE Annual Conference and Exposition, Chicago, Illinois.

Khun, D., Iordanou, K., Pease, M., y Wirkala, C. (2008). Beyond control of variables: What needs to develop to achieve skilled scientific thinking? [Más allá del control de variables: ¿Qué se necesita desarrollar para lograr habilidades de pensamiento científico]. Cognitive development, 23, 435-451.

Lewis, M. D. (2005). Arguing in English and French asynchronus online discussion [Argumentando en Inglés y Francés dentro de discusiones asincrónicas en línea]. Journal of pragmatics, 37, 1801-1818.

Mason, L., y Scirica, F. (2006). Prediction of students' argumentation skills about controversial topics by epistemological understanding [Predicción de las habilidades argumentativas de estudiantes por las posturas epistemológicas en temas controversiales]. Learning and Instruction, 16(5) 492-509.

Nussbaum, E. M, Sinatra G. M., y Poliquin, A., (2008). Role of epistemic beliefs and scientific argumentation in science learning [El rol de las creencias epistémicas y argumentación científica en el aprendizaje de la ciencia]. International Journal of Science Education, 30(15), 1977-1999.

Oakleaf, M. (2009). Using rubrics to assess information literacy: an examination of methodology and interrater reliability [El uso de rúbricas para evaluar literacidad informativa: una examinación de la metodología y la confiabilidad interna]. Journal of the American Society for Information Science and Technology, 60(5), 969-983

Penny L., y Murphy E., (2009). Rubrics for designing and evaluating online asynchronous discussions [Rúbricas para diseñar y evaluar discusiones asincrónicas en línea]. British Journal of Educational Technology, 40 (5), 804-820.

Roegiers, X. (2006). Approche par compétences dans l'enseignement supérieur et cadre européen de qualifications: opportunités, enjeux et dérives [Aproximación por competencias en la enseñanza de educación superior y la catedra europea de calidad: oportunidades, apuestas y desviaciones]. Conferencia magistral del Coloquio La logique des compétences: chance ou danger? Vers un cadre de qualification dans l'enseignement Supérieur. París, Francia. Recuperado de http://www.bief.be/index.php?found=1\&lg=fr\&rs=22\&s=4\&uid=34

Sadler, T. y Fowler, S., (2006). A threshold model of content knowledge transfer for socioscientific argumentation [Un modelo del umbral de conocimiento del contenido transferencia para la argumentación sociocíentifica]. WileyPeriodicals, Recuperado de www.interscience.wiley.com

Tobón, S.; Rial, A.; Carretero M.A. y García, J.A. (2006). Competencias, calidad y educación superior. Recuperado de http://books.google.co.cr/books?id=jW7G7qRhry4C\&printsec=front cover\&dq=Tob\%C3\%B3n, + S. $+\% 282006 \% 29 .+$ Booksgoogle\&hl=es\&sa=X\&ei=uRsDUvLdFor Q9gTtpoDoBA\&ved=0CC4Q6AEwAA\#v=onepage\&q\&f=false

Veerman, A. L., Andriessen, J. E., y Kanselaar, G. (2000). Learning through syncronus electronic discussion [Aprendizaje a través de la discusión asincrónica en línea].. Computer \& Education, 34(3-4), 269-290. 
Welch, H. Suri, D., y Durant, E. (2009). Rubrics for assessing oral communication in the capstone design experience: Development, application, analysis and refinement [Rúbricas para evaluar la comunicación oral el toque de la experiencia de diseño: desarrollo, aplicación, analisis y refinamiento]. International Journal of Engineering Education, 25(5), 952-961.

Ying-Hua, G., Chin-Chung y Fu-Kwun (2006). Content analysis of online discussion on a seniorhigh-school discussion forum of a virtual physics laboratory [Análisis de contenido de discusiones en línea dentro de foros de discusión en línea en un laboratorio virtual de física].. Instructional Science, 34(4): 279-311.Material complementario

\section{Rúbrica para evaluar mensajes en un foro de discusión.}

Introducción. El ser capaz de argumentar las ideas que se sustentan permite muchos éxitos en la vida, por ello en la formación profesional hoy en día se tienen diferentes estrategias para apoyar el desarrollo de esta competencia.

En la siguiente tabla se muestran elementos del mensaje que pueden te pueden apoyar para elaborar argumentos más sólidos en foros de discusión, además puede ayudar a valorar tus participaciones ya realizadas en otros foros.

Instrucciones. La tabla está dividida en 4 columnas (elementos del mensaje y 3 niveles de desempeño para cada elemento de la columna uno; donde 1 es básico, 2 es intermedio y 3 avanzado).

Tu puedes evaluar antes o después de haber subido tus argumentos en el foro marcando el nivel de cada elemento (lenguaje, colaboración, respeto, fundamentación, contraargumentación).

\begin{tabular}{|l|l|l|l|}
\hline \multirow{2}{*}{$\begin{array}{c}\text { Elementos del } \\
\text { mensaje }\end{array}$} & \multicolumn{3}{|c|}{ Características (niveles de desempeño) } \\
\cline { 2 - 4 } & \multicolumn{1}{|c|}{1} & \multicolumn{1}{|c|}{2} \\
\hline Lenguaje. & $\begin{array}{l}\text { Se utilizan de manera superficial los } \\
\text { conceptos clave en los mensajes. }\end{array}$ & $\begin{array}{l}\text { Se hace referencia escasa de los conceptos } \\
\text { clave. }\end{array}$ & $\begin{array}{l}\text { Se hizo referencia amplia de los conceptos } \\
\text { clave. }\end{array}$ \\
\hline Colaboración. & $\begin{array}{l}\text { Al escribir los mensajes busca } \\
\text { informar de la postura a los } \\
\text { compañeros. }\end{array}$ & $\begin{array}{l}\text { Al escribir los mensajes busca convencer a } \\
\text { los compañeros tomando en cuenta sus } \\
\text { contribuciones. }\end{array}$ & $\begin{array}{l}\text { Al escribir los mensajes busca llegar a acuerdos } \\
\text { con los compañeros, al retroalimentar los } \\
\text { puntos de vista y buscar consenso. }\end{array}$ \\
\hline Respeto. & $\begin{array}{l}\text { Rara vez se trata de comprender las } \\
\text { aportaciones de los compañeros pero } \\
\text { evita descalificar. }\end{array}$ & $\begin{array}{l}\text { Por lo regular se trata de comprender las } \\
\text { aportaciones de los compañeros } \\
\text { respondiéndoles sin descalificarlos. }\end{array}$ & $\begin{array}{l}\text { Frecuentemente se trata de comprender las } \\
\text { aportaciones de los compañeros valorando sus } \\
\text { participaciones al respoderles. }\end{array}$ \\
\hline Fundamentación. & $\begin{array}{l}\text { Se hacen afirmaciones basadas en } \\
\text { razones que todos conocen. }\end{array}$ & $\begin{array}{l}\text { Se hacen afirmaciones basadas en } \\
\text { experiencias personales que demuestran su } \\
\text { validez. }\end{array}$ & $\begin{array}{l}\text { Se hacen afirmaciones basadas en fuentes } \\
\text { científicas o de evidencia empírica (entrevistas, } \\
\text { conferencias, artículos, etc.). }\end{array}$ \\
\hline Contraagumentación & $\begin{array}{l}\text { Se dan contraargumentos a los } \\
\text { compañeros tomando en cuenta de } \\
\text { forma muy general lo que han } \\
\text { comentado. }\end{array}$ & $\begin{array}{l}\text { Se dan contraargumentos a los compañeros } \\
\text { valorando lo que han comentado. }\end{array}$ & $\begin{array}{l}\text { Se dan contraargumentos a los compañeros } \\
\text { haciendo referencia a lo expuesto por ellos y } \\
\text { señalándoles fortal ezas y debilidades en su } \\
\text { argumento. }\end{array}$ \\
\hline
\end{tabular}

\section{Glosario}

Lenguaje: Manejo apropiado y amplio de los conceptos claves de la temática que se argumenta. 
Colaboración: Contribuir a lograr acuerdos a partir de la deliberación en el foro.

Respeto: Tomar en consideración las participaciones de los compañeros.

Fundamentación: Manejo de evidencias alrededor de una afirmación.

Contraargumentación: Es una réplica que toma de base el mensaje de otro un compañero. 